\title{
Comment on "AC frequency characteristics of coplanar impedance sensors as design parameters" by Jongin Hong, Dae Sung Yoon, Sung Kwan Kim, Tae Song Kim, Sanghyo Kim, Eugene Y. Pak and Kwangsoo No, Lab Chip, 2005, 5, 270
}

\section{Pontus Linderholm and Philippe Renaud}

First published as an Advance Article on the web 13th October 2005

DOI: $10.1039 / \mathrm{b512077b}$

In their article, "AC frequency characteristics of coplanar impedance sensors as design parameters", Hong et al. ${ }^{1}$ present an analytical model for the calculation of the resistance between two electrodes in a microchannel. However, their measurements fit poorly with their suggested model, and they attribute the discrepancies to electrode thickness and a 'fringing effect'. In this note we show that the discrepancies are due to the neglected channel height in their model, and that by including two additional transformations, the resistance between two electrodes in a microchannel can be accurately modelled.

The cell constant describes the proportionality between the resistivity of the medium in contact with two electrodes and the resulting resistance. The usefulness of the cell constant for microelectrode design optimization has been presented previously. ${ }^{2,3}$ Both these papers treat sensor design for coplanar strip lines in contact with a semi-infinite medium. In order to use the Schwarz-Christoffel (SC) conformal mapping, as described in these papers, the electrodes must be symmetrical about the origin, and the electrode width must be negligible compared to the sample height.

In the article by Hong et al., ${ }^{1}$ the geometry is such that the height of the channel $(30 \mu \mathrm{m})$ is not negligible compared to the electrode widths and spacings $(20-500 \mu \mathrm{m})$ of their device. The approximation of a semi-infinite medium on top of the electrodes therefore fails, since it does not take into account the insulating boundary condition at the top of the channel.

We will show in this paper that the large discrepancies between theory and experiment presented by Hong et al. (see Fig. 8 and Fig. 10 in their paper) can be reconciled by

Microsystems Laboratory, Swiss Federal Institute of Technology, Lausanne, 1015, Switzerland

a)

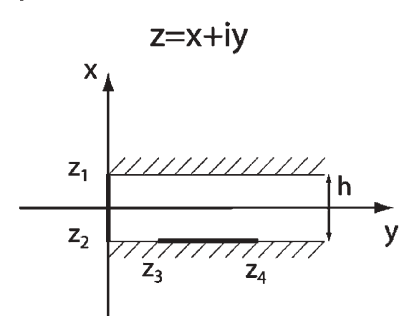

b)

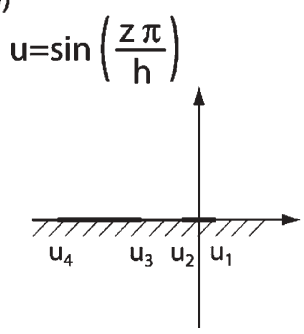

appropriate use of conformal mapping for calculation of the cell constant. We introduce two additional conformal mappings that must be performed before the resistance can be analyzed as suggested by Hong. These two conformal mappings are a sinetransformation followed by a bilinear transformation.

If we consider the $2 \mathrm{D}$ case, the length-wise cross-section of the microchannel can be modelled as a rectangle (Fig. 1(a)). Exploiting the symmetry of the geometry, we can replace the left half of the microchannel with a conductor at the center between the two electrodes. The problem is then to calculate the resistance between the center conductor and one of the active electrodes.

Let the geometry of the real microchannel be defined in the $Z$-plane, with coordinates

$$
z=x+\mathrm{i} y
$$

The center conductor is defined by the points $z_{1}=h / 2$ and $z_{2}=-h / 2$, and the microelectrode is defined by $z_{3}=-h / 2+$ $\mathrm{i} s / 2$ and $z_{4}=-h / 2+\mathrm{i}(s / 2+w)$, where $s$ is the interelectrode distance, $w$ is the electrode width and $h$ is the height of the channel (Fig. 1(a), note that the axes are reversed).

The problem now consists of transforming the rectangle, with the symmetry conductor at the left side and the active electrode along the bottom wall, to a half-plane with all channel walls and the symmetry electrode along the real axis. This can be done using a sine-transformation of the $Z$-plane. Using the transformation of a trigon, as described by Schinzinger, ${ }^{4}$ the first transformation of the microchannel should be:

Since

$$
u=\sin \left(\pi \frac{z}{h}\right)
$$

$$
\sin (x+\mathrm{i} y)=\sin (x) \cosh (y)+\mathrm{i} \cos (x) \sinh (y)
$$

c)
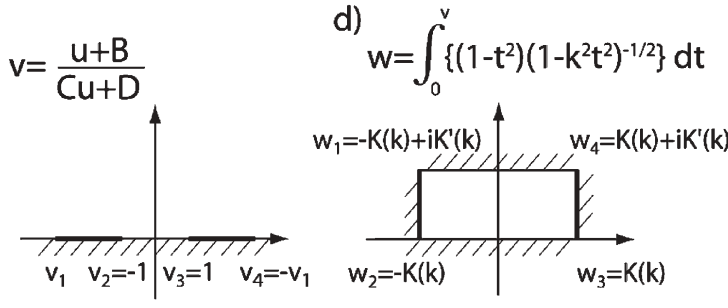

Fig. 1 (a) A cross-section of half of the microchannel, with the center conductor and one of the electrodes marked in bold. To use the SchwarzChristoffel transform, the geometry of the microchannel must first go through two complex transformations: (b) the sine-transform to place the channel walls on the real axis of the $U$-plane, followed by (c) the bilinear transform to make the electrodes symmetrical about the origin. (d) Finally, the SC mapping can be used to obtain a geometry in which the resistance can be easily calculated. 
all points on the real axis in $Z$ will lie on the real axis in $U$, as will all points along the walls of the microchannel $\left(z= \pm \frac{h}{2}+\mathrm{i} y\right)$. The points in the interior of the right half of the channel are mapped to the top half-plane in the $U$-plane (Fig. 1(b)).

To be able to use the SC conformal mapping as described by Jacobs et al., the electrodes must be of equal length and symmetrical about the origin. This is not yet the case in the $U$-plane. We therefore need to perform an asymmetric "stretching" of this plane, which can be carried out using a bilinear transformation: ${ }^{5}$

$$
v=\frac{u+B}{C u+D}
$$

The constants $B, C$ and $D$ must be adjusted so that the electrodes are symmetric around the origin and of equal width. We also set $v_{3}=-v_{2}=1$, so that we can proceed directly with the SC mapping afterwards. This gives us a system of equations

$$
\left\{\begin{array}{l}
\frac{u_{1}+B}{C u_{1}+D}=-\frac{u_{4}+B}{C u_{4}+D} \\
\frac{u_{2}+B}{C u_{2}+D}=-1 \\
\frac{u_{3}+B}{C u_{3}+D}=1
\end{array}\right.
$$

Solving the systems of equations for $B, C$ and $D$ then gives the following expression for $v_{4}$ :

$v_{4}=$

$$
\frac{\left(u_{3}-u_{2}\right)\left(u_{4}-u_{1}\right)}{u_{4}\left(u_{3}+u_{2}-2 u_{1}\right)+2 \sqrt{\left(u_{4}-u_{3}\right)\left(u_{4}-u_{2}\right)\left(u_{3}-u_{1}\right)\left(u_{2}-u_{1}\right)}+u_{1} u_{2}+u_{3}\left(-2 u_{2}+u_{1}\right)}
$$

For the geometry in the $V$-plane, the modulus for the SC transformation is given by ${ }^{4}$

$$
k_{\mu \text { channel }}=\frac{1}{v_{4}}
$$

where $u_{1} . . u_{4}$ are the coordinates of the electrodes in the $U$-plane. Since the cell constant calculated using the procedure described here only represents half of the microchannel, we need to multiply the expression for the cell constant in Olthuis et al. ${ }^{3}$ by a factor 2 . The final expression for the cell constant in a microchannel is then:

$$
\kappa_{\mu \text { channel }}=4 \frac{K\left(k_{\mu \text { channel }}\right)}{K^{\prime}\left(k_{\mu \text { channel }}\right)}
$$

where $K$ and $K^{\prime}$ are elliptic integrals of the first order, and can be calculated using a commercial software package such as Matlab ${ }^{\mathbb{R}}$.

In the paper by Hong et al., the bulk resistances of different geometries and different conductivities have been measured, and the cell constants subsequently calculated. However, the resistivities of the different solutions are not given. Furthermore, using the only given resistivity, that of the deionized (DI) water, their cell constants do not correspond to the measured values. For example, the value of the bulk resistance through DI water can be deduced from Fig. 6 in Hong's paper, and it is found to be $\sim 20 \mathrm{M} \Omega$. The authors claim that the conductivity of this DI water is $0.06 \mu \mathrm{S} \mathrm{cm}^{-1}$. According to eqn (8) in their paper, the cell constant should then be $\sim 0.01$

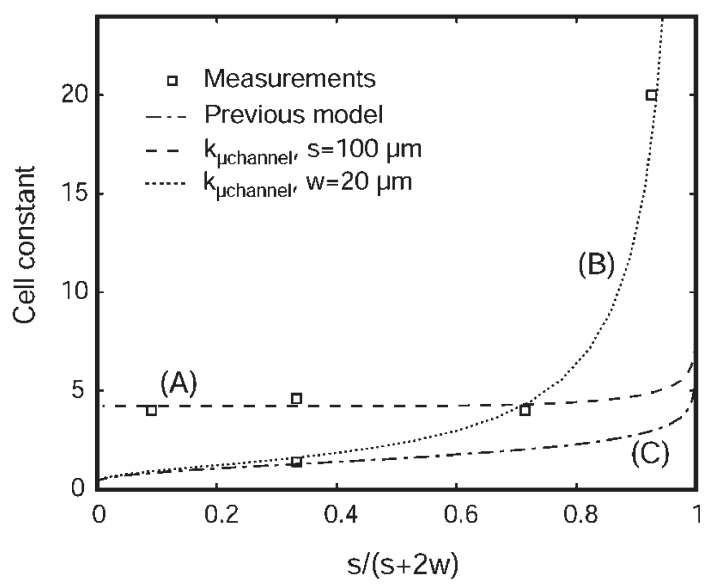

Fig. 2 Cell constants for different geometries with a channel height of $30 \mu \mathrm{m}$. The experimental data points are taken from Fig. $8 \mathrm{a}$ and Fig. 10a in Hong's paper, assuming a conductivity of $20 \mu \mathrm{S} \mathrm{cm}{ }^{-1}$. Lines A and B represent the theoretical values for the cell constants developed in this paper, A for the case of a fixed electrode width $(w)$, and B for a fixed gap $(s)$. Line $\mathrm{C}$ represents the cell constants for a semi-infinite sample, as described by Hong et al.

assuming an electrode length of $100 \mu \mathrm{m}$. This is two orders of magnitude lower than the theoretical value they present.

To be able to compare their measurements with our theory, we have therefore assumed that the indicated resistivity of the DI water is not correct. We believe this is reasonable, since the resistivity of ultra-pure water quickly drops due to contaminations, and Hong et al. do not report having made any reference measurements of the resistivities. We took the liberty of fitting their measurements to our model by varying the conductivity. We then found a more likely value to be $20 \mu \mathrm{S} \mathrm{cm}{ }^{-1}$. In Fig. 2 we indicate the cell constants of Hong et al., after having adjusted the conductivities, and the theoretical results based on our model. The model then provides a very good fit with their measurements.

In conclusion, we have presented a model for the calculation of the cell constants for coplanar electrodes in a microchannel in which the height of the channel is taken into account. The cell constants are easily calculated by first finding the values of $u_{1} \ldots u_{4}$ (eqn (2)), and then use these four values to find the modulus (eqns (6) and (7)) for the elliptic integrals and finally evaluate the elliptic integrals of eqn (8) using Matlab ${ }^{\circledR}$, Mathematica ${ }^{\circledR}$, or any other suitable software. Using this model, we can explain the measurements obtained by Hong et al., and we therefore argue that our model is more suitable for lab-on-chip applications.

\section{References}

1 J. Hong, D. S. Yoon, S. K. Kim, T. S. Kim, S. Kim, E. Y. Pak and K. No, Lab Chip, 2005, 5(3), 270-279.

2 P. Jacobs, A. Varlan and W. Sansen, Med. Biol. Eng. Comput., 1995, 33, 6, 802-810.

3 W. Olthuis, W. Streekstra and P. Bergveld, Sens. Actuators, B, 1995, 24, 1-3, 252-256.

4 R. Schinzinger and P. A. A. Laura, Conformal mapping: methods and applications, 1991, Elsevier, Amsterdam, p. 60.

5 Z. W. Du, J. S. Fu and K. Gong, Int. J. Infrared Millimeter Waves, 1999, 20, 2, 351-356. 